\title{
Correlation Electron Cyclotron Emission diagnostic in TCV
}

\author{
M. Fontana, ${ }^{1, a)}$ L. Porte,${ }^{1}$ and P. Molina Cabrera ${ }^{1}$ \\ Swiss Plasma Center, EPFL, Lausanne, 1015, Switzerland
}

(Dated: 13 March 2018)

The correlation electron cyclotron emission (CECE) diagnostic of TCV has recently been upgraded. It now has the choice of three lines of sight: two horizontal lines placed on the low field side of the vessel, perpendicular to the magnetic field, and a dual-axis steerable antenna. The polarization of the radiation collected by the latter can be rotated using a universal polarizer situated in the transmission line. This line is also shared with a reflectometry system, allowing simultaneous measurements of temperature and density fluctuations in the same plasma volumes. When using this line it is possible to choose between two dedicated front ends characterized by different local oscillator frequencies, adding flexibility in the choice of the plasma region to be studied. The intermediate frequency section is now equipped with six frequency tunable YIG filters allowing the study of turbulence properties in a wide range of radial positions. When studying fluctuations over the whole video bandwidth the minimum detectable fluctuation level is $\delta T_{e} / T_{e} \sim 0.5 \%$. The new system has been used to measure electron temperature fluctuations over a large fraction of the plasma profiles in a series of plasmas with triangularity varying from 0.6 to -0.6 but comparable collisionality profiles.

\section{INTRODUCTION}

In magnetically confined plasmas the measured particle and energy fluxes exceed those calculated from neoclassical transport theory by at least an order of magnitude. This anomalous transport is generally considered to be due to multi-field microfluctuations such as those that are generated by turbulent phenomena. To study these phenomena it is necessary to measure broadband, low amplitude fluctuations of various quantities such as electron density, electron temperature and electric field.

One of the available choices to measure electron temperature fluctuations is the Correlation Electron Cyclotron Emission (CECE) diagnostic. In a standard ECE diagnostic the minimum detectable fluctuation level is limited by:

$$
\left(\frac{\delta T_{e}}{T_{e}}\right)^{2} \sim \frac{B_{v i d}}{B_{I F}}
$$

where $B_{I F}$ is the radio frequenqy ( $\mathrm{RF}$ ) bandwidth and $B_{v i d}$ is the signal bandwidth at the acquisition. This value is usually too large to properly study microfluctuations in tokamaks, whose relative amplitudes are often of the order of $1 \%$. The CECE principle consists of using cross-correlation analysis on signals coming from different channels ${ }^{1}$ that are observing the same fluctuating structures: thermal noise, uncorrelated in the two signals, is suppressed, highlighting the fluctuations common to the two channels. By computing the cross correlation coefficient between these two signals it is possible to obtain spatial information on the structures of interest, such as an estimate of the radial correlation length. If correlation analysis is performed over $\mathrm{N}$ time samples, then the minimum detectable fluctuation is reduced to:

\footnotetext{
a)matteo.fontana@epfl.ch
}

$$
\left(\frac{\delta T_{e}}{T_{e}}\right)^{2} \sim \frac{1}{\sqrt{N}} \frac{B_{v i d}}{B_{I F}}
$$

Over the years different diagnostic setups have been used to perform this kind of analysis: a review can be found in ${ }^{1}$. The most common, used also in TCV, is based on spectral decorrelation of noise: different channels are set at frequencies that keep them separated in physical space but close enough to detect the same fluctuating structures.

The Tokamak à Configuration Variable (TCV) is a medium size tokamak whose main assets are an unparalleled shaping flexibility, a high power electron heating and current drive, based on gyrotron sources, and a recently installed neutral beam system for direct ion heating ${ }^{2}$. Its CECE system, detecting emission at the second harmonic of the extraordinary mode (X2), has been recently upgraded with six new YIG frequency tunable filters, two dedicated, alternative front ends and a steerable, high resolution line of sight that is shared with a reflectometry system. This new setup has been used to obtain measurements of fluctuation amplitudes in the range $0.35<\rho_{\psi}<0.9$ for a variety of shaped plasmas with triangularity varying from 0.6 to -0.6 , where $\rho_{\psi}$ is the radial coordinate defined as $\sqrt{\left(\psi-\psi_{\text {axis }}\right) /\left(\psi_{\text {axis }}-\psi_{\text {edge }}\right)}$ where $\psi$ is the poloidal field flux, $\psi_{\text {axis }}$ is the flux at the magnetic axis position and $\psi_{\text {edge }}$ is the flux at the last close flux surface..

\section{CORRELATION ECE IN TCV}

The CECE system originally installed in TCV in $2007^{3}$ has been substantially upgraded. A new steerable line of sight, shared with a reflectometry system, is now connected to dedicated radiofrequency (RF) sections. The intermediate frequency (IF) section has been expanded 


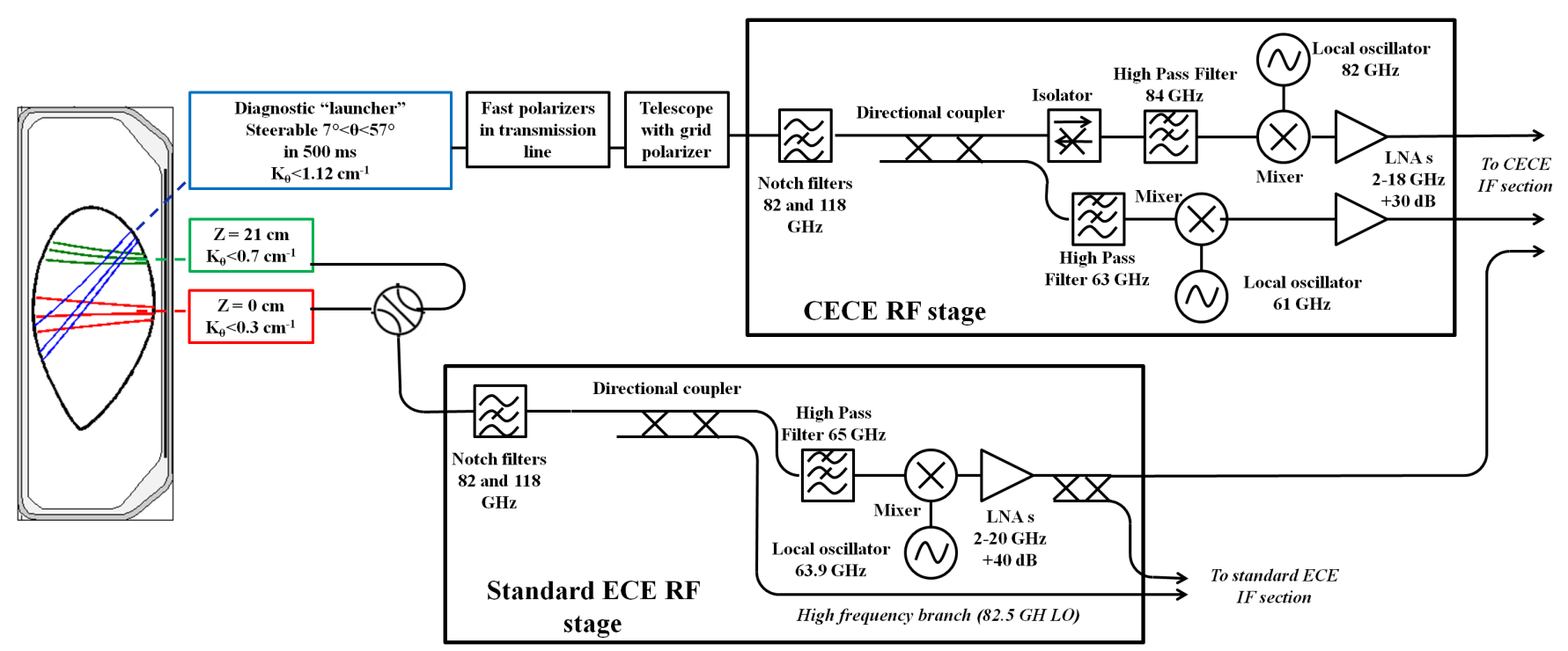

FIG. 1. Summary of the CECE system in TCV from the vessel to the RF sections of the radiometers.

to six channels, each containing a frequency tunable yttrium iron garnet (YIG) filter. The main goal of the new system is to study electron temperature fluctuations in a large section of the radial profile and in a variety of plasma configurations, exploiting the flexibility provided by the multiple lines of sight and the possibility of precisely choosing the measurement location for each channel in every discharge.

\section{A. Lines of sight and radiometers}

A schematic representation of the different lines of sight available to the CECE in TCV and the corresponding radio frequency sections are presented in figure 1 . The CECE system can use three different lines of sight. The first two are on the low field side (LFS), perpendicular to the magnetic field, one situated at the vessel midplane $(z=0)$ the other at $z=21 \mathrm{~cm}$ above it. The equatorial line employs a Teflon lens focusing the emitted radiation into a circular $2.5 \mathrm{~cm}$ waveguide. The $z=21$ $\mathrm{cm}$ line instead uses a Gaussian horn and an elliptical mirror to focus radiation into the waveguide. The $z=0$ and $z=21 \mathrm{~cm}$ antennas are sensitive to modes with $k_{\theta}<0.4 \mathrm{~cm}^{-1}$ and $k_{\theta}<0.3 \mathrm{~cm}^{-1}$ respectively $^{4}$, where $k_{\theta}$ is the poloidal wave number. The choice of the viewing line is made using a remotely controlled waveguide switch. These horizontal lines carry radiation to the standard ECE radiometer.

This radiometer has two $\mathrm{RF}$ sections with different $\mathrm{LO}$ frequencies. The power going into the lower frequency branch (63.9 GHZ LO) is split in two by a directional coupler after the mixing stage: half of it goes into the standard ECE channels while the other half can be directed to IF section of the CECE.

\section{Steerable antenna}

The third line of sight available to the CECE system on TCV is based on a recently installed steerable antenna. Its schematics are shown in figure 2. The antenna is located on the LFS wall and perpendicular to it, in the upper part of the vessel, $\mathrm{z}=45 \mathrm{~cm}$ above the chamber mid plane. Its line of sight can be moved by changing the angle of the last mirror ( $\theta$ in figure 2 and by rotating the whole antenna body around its axis (angle $\phi$ in figure 2). $\theta$ can be varied between 7 and $55^{\circ}$ with respect to the antenna axis, while $\phi$ can vary between -90 and $90^{\circ}$ with respect to the plane perpendicular to the LFS vessel wall. This allows one to accurately select the measurement location in all plasma configurations in TCV and, additionally, to perform oblique ECE measurements ${ }^{5}$. The effective vertical size of the emission volumes observed with this line strongly depends on $\theta$, the selected channels frequencies and the plasma position. In optimal conditions it can resolve modes with $k_{\theta}<1.12 \mathrm{~cm}^{-1}$.

The collected radiation is transmitted to the CECE and the reflectometer through a HE11 $63.5 \mathrm{~mm}$ inner diameter corrugated waveguide. To guarantee optimal coupling between the emitted radiation and the diagnostic systems in all discharges, the transmission line contains two remotely controlled polarizers, one linear and one elliptical, whose positions can be varied during a discharge $\left(0.1 \mathrm{~s} \text { for } 90^{\circ} \text { polarization rotation }\right)^{6}$. Given a reference discharge it is possible to calculate the optimal polarizers setting that would grant the best coupling to the two diagnostics for the selected line angles ${ }^{7}$. The transmission line is coupled to the diagnostics through a telescope, shown in figure 3. A pair of elliptical mirrors direct the radiation to a grid polarizer that separates vertical and horizontal polarizations. The two halves of the incoming signal are directed to two gaussian horns, respectively 


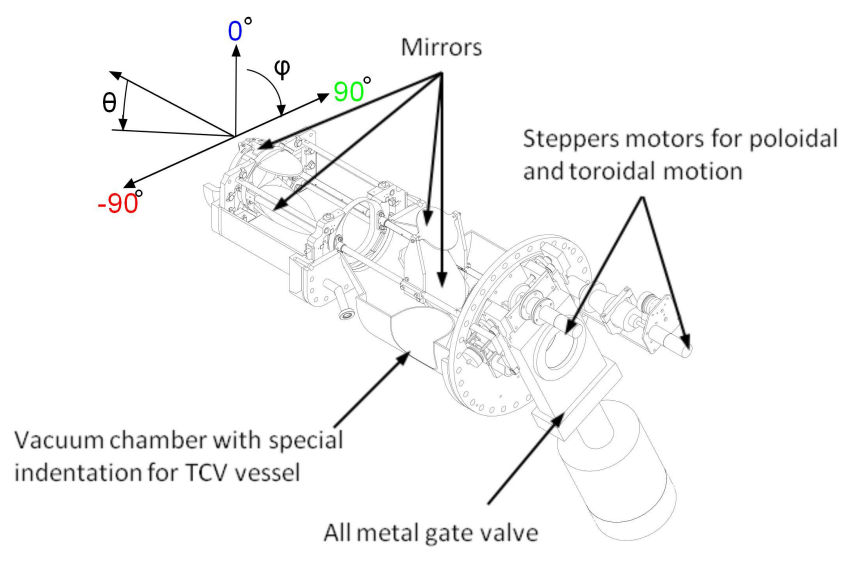

FIG. 2. Schematic of the steerable line of sight connected to the CECE and reflectometry systems in TCV. Its line of sight can be rotated over $\theta$ and $\phi$.

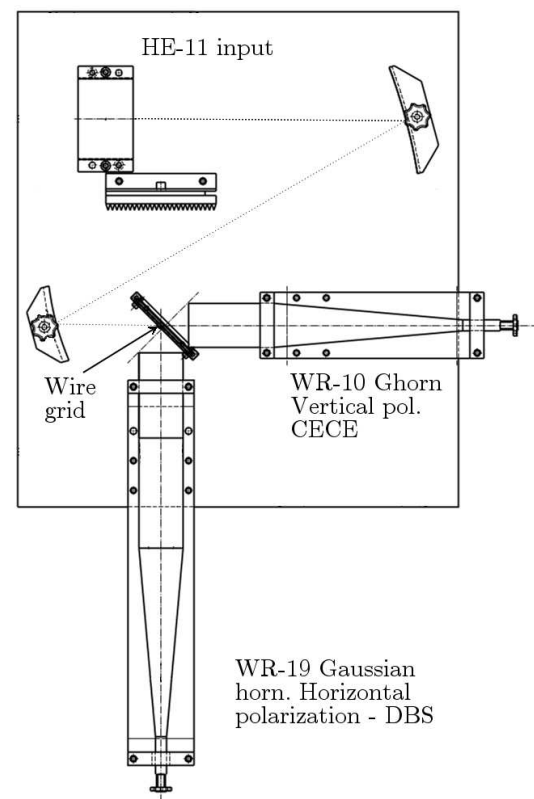

FIG. 3. Schematics of the telescope coupling the power gathered by the steerable line of sight to the CECE and the DBS reflectometer.

connected to WR-10 and WR-15 rectangular waveguides. The former is connected to the CECE RF section. That is composed by two separate front ends with different local oscillators (working at $61 \mathrm{GHz}$ and $82 \mathrm{GHz}$ respectively). The mixing stage in use can be switched in between discharges.

The WR-15 horn is instead connected to an O-mode reflectometer. The presence of this system in parallel with the CECE allows the design of discharges where simultaneous measurements of temperature and density fluctuations in the same plasma volumes can be performed. By applying correlation analysis techniques to such signals it is possible to calculate the $\mathrm{n}-\mathrm{T}$ fluctuations crossphase.

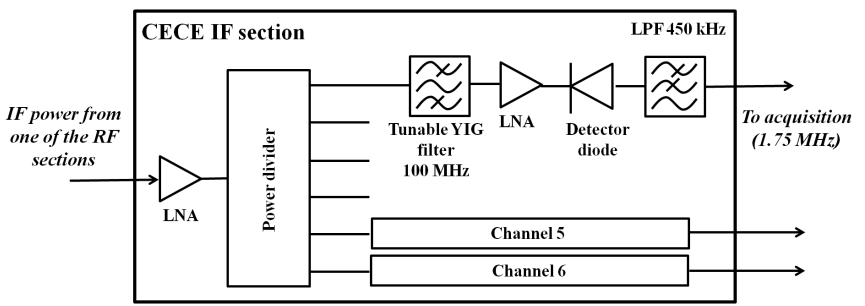

FIG. 4. Schematics of the IF section of the CECE diagnostic on TCV.

This measurement is of particular interest since it relates directly to the turbulent transport terms and can be compared with the predictions of gyrokinetic simulations ${ }^{8}$.

\section{B. Intermediate frequency section}

The downconverted radiation coming from the $\mathrm{RF}$ section chosen for a particular discharge is passed into the CECE IF section with flexible RF cables. There it is amplified with a $30 \mathrm{~dB}$ low noise amplifier (LNA) connected to a six way power divider that splits the signal into six identical channels. Each of these consists of an YIG filter, an additional amplification stage (20 dB LNA) and finally a schottky diode for rectification. The filters are OMNYIG M2806DD. They have $-3 \mathrm{~dB}$ bandwidth of $100 \mathrm{MHz}$ and their center frequencies are independently tunable with a 12 bit digital controller, in the $6-18 \mathrm{GHz}$ range. The signals are then low pass filtered in a fifth order butterworth at $450 \mathrm{kHz}$ and amplified with electronically controlled gain before being acquired at 1.75 $\mathrm{MHz}$. With these parameters, looking at fluctuations over the whole $450 \mathrm{kHz}$ band, the minimum detectable fluctuation when correlation analysis is performed over $100 \mathrm{~ms}$ period is $\delta T_{e} / T_{e} \sim 0.33 \%$ according to equation 2. Schematics of the CECE IF sections are shown in figure 4 .

\section{EXAMPLES OF MEASUREMENTS}

In 2013, the new six channels IF section of the CECE system has been used to study the radial profile of electron temperature fluctuations in limited deuterium plasmas with varying triangularity from 0.4 to $-0.4^{9}$. The discharges were performed at $\mathrm{z}=0$, using the LFS line of sight and the corresponding radiometer RF stage. Apart from the varying shape the other plasma characteristics were kept constant: $1.44 \mathrm{~T}$ of axial magnetic field, $1.15 * 10^{19} \mathrm{~m}^{-2}$ line integrated density, plasma current of $220 \mathrm{kA}$ and ohmic heating only. Discharges with the same shape were repeated multiple times moving the CECE IF frequencies from a discharge to another to gather measurements in multiple radial locations. The system was able to measure fluctuations below $1 \%$. It was found that the level of fluctuation decreases with 


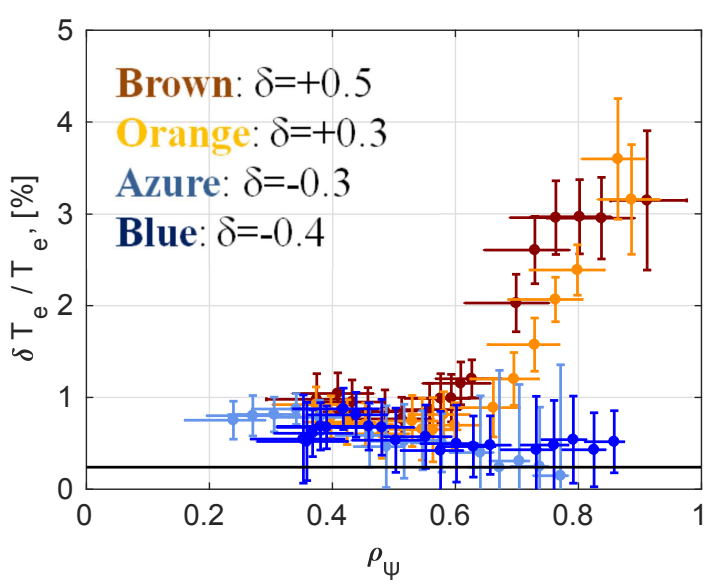

FIG. 5. Radial profiles of relative electron temperature fluctuation amplitude for different triangularity values. The fluctuations amplitude is reduced changing shape from positive to negative triangularity and the effects extends up to $\rho_{\psi}=0.65$.

triangularity going from positive to negative over the $0.35<\rho_{\psi}<0.8$ range. In particular the fluctuations suppression was observed also for radial positions closer to the plasma core where the local value of triangularity is strongly reduced. Moving towards the plasma interior, in fact, the flux surfaces become more and more circular and the effects of edge shaping should become marginal.

In 2016, after the connection of the new steerable line of sight and the new dedicated RF sections for the CECE, these measurements have been repeated with the aim of exploiting the wider frequency range now available to measure fluctuations over a larger fraction of the radial profile, in particular towards the outer core. The discharges have been repeated at four different triangularity values: $-0.4,-0.3,0.3$ and 0.5, at $\mathrm{z}=30 \mathrm{~cm}$ to optimize the spot size of the upper lateral line of sight. The new measurements are in agreement with those taken with the horizontal line of sight but further extend the accessible range to $0.3<\rho_{\psi}<0.95$. The application of cross-correlation between neighbouring channels allows the detection of relative fluctuations smaller than $1 \%$ but still above the expected noise floor for the $500 \mathrm{~ms}$ average time used here $(0.22 \%)$. As expected, the change in shape from positive to negative triangularity strongly reduces the amplitude of the measured electron temperature fluctuations. This effect is strongest at $\rho_{\psi}=0.9$, where relative fluctuations are reduced from $3.5 \%$ to less than $1 \%$ but it is visible all the way up to $\rho_{\psi}=0.65$.

\section{CONCLUSIONS}

The upgraded CECE diagnostic of TCV can observe the plasma through three diferent lines of sight: two perpendicular to the magnetic field on the LFS, respectively at $z=0$ and $z=21 \mathrm{~cm}$, and one upper lateral, steerable line of sight. The power collected by the latter is split by a grid polarizers between the CECE and a reflectometer system. The transmission line connected to this steerable antenna contains two polarizers that allow one to optimize the coupling between the collected radiation and the two diagnostics. The same transmission line carries power also to a reflectometer that, in the future, could be used together with the CECE to take simultaneous measurements of electron temperature and density fluctuations in the same plasma volume and compare their phase. The IF stage of the CECE now contains six independently tunable, narrow band, YIG filters that allow one to accurately choose the emission volumes for each channel in a wide range of radial positions. The flexibility of the system, combined with the innate versatility of TCV in obtaining a wide range of plasma shapes in a number of different positions in the vessel, make the CECE diagnostic able to observe an extended fraction of the plasma poloidal section.

The system has been exploited with a series of plasmadischarges in which the effects of triangularity on electron temperature relative fluctuation amplitude has been studied in the range $0.3<\rho_{\psi}<0.9$. The system proved able to measure fluctuations with relative amplitudes well below 1\%. A strong reduction in the fluctuations relative amplitude for $\rho_{\psi}>0.7$ was observed in negative triangularity discharges with respect to positive triangularity.

\section{ACKNOWLEDGMENTS}

This work has been carried out within the framework of the EUROfusion Consortium and has received funding from the Euratom research and training programme 2014-2018 under grant agreement No. 633053. The views and opinions expressed herein do not necessarily reflect those of the European Commission. This project has received funding from the HGF Virtual Institute on Plasma Dynamical Processes and Turbulence Studies using Advanced Microwave Diagnostics. This work was also supported in part by the Swiss National Science Foundation.

${ }^{1}$ C. Watts, Fusion Science and Technology 52, 176 (2007).

${ }^{2}$ S. Coda and T. C. V. Team, Nucl. Fusion 55, 104004 (2015).

${ }^{3}$ V. S. Udintsev, G. Turri, E. Asp, C. Schlatter, T. P. Goodman, O. Sauter, H. Weisen, P. Blanchard, S. Coda, B. P. Duval, E. Fable, A. Gudozhnik, P. F. Isoz, M. A. Henderson, I. Klimanov, X. Llobet, P. H. Marmillod, A. Mueck, L. Porte, and H. Shidara, Fusion Science and Technology 52, 161 (2007).

${ }^{4} \mathrm{R}$. Bravenec and A. Wootton, Review of Scientific Instruments 66, 802 (1995).

${ }^{5}$ T. P. Goodman, V. S. Udintsev, I. Klimanov, A. Mueck, O. Sauter, and C. Schlatter, Fusion Science and Technology 53, 196 (2008).

${ }^{6}$ M. Silva, T. Goodman, F. Felici, and L. Porte, Fusion Engineering and Design Proceedings of the 26th Symposium of Fusion Technology (SOFT-26), 86, 1256 (2011).

${ }^{7}$ F. Felici, "ECPOL: equations and Matlab tools for EC wave reflection and polarization calculations," Tech. Rep. 174984 (EPFLCRPP, 2012).

${ }^{8}$ A. E. White, W. A. Peebles, and T. L. Rhodes, Physics of Plasmas 17, 056103 (2010). 
${ }^{9}$ L. Porte et al., in Proceedings of 25th IAEA Fusion Energy Con- $\quad$ ference, St Petersburg, Russia (2014). 